Title:

Author(s):

Submitted to:
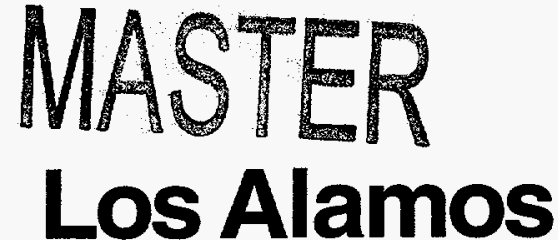

NATIONAL LABORATORY
Separations Technology Development to Support Accelerator-Driven Transmutation Concepts

RECEIVED

OCT 11 19SS

OSTI

Francesco Venneri, LER

Edward Arthur, NMSM

Charles Bowman, LER

Roberto Camassa, T-7

Robert Ecke, MST-10

Reed Jensen, CST

Ning Li, MST-10

Brian Newnam, LER

Mark Williamson, NMT-6

DOE Office of Scientific and Technical

Information (OSTI)

JDISTRIBUTION OF THIS DOCUMENT IS UNLIMITED

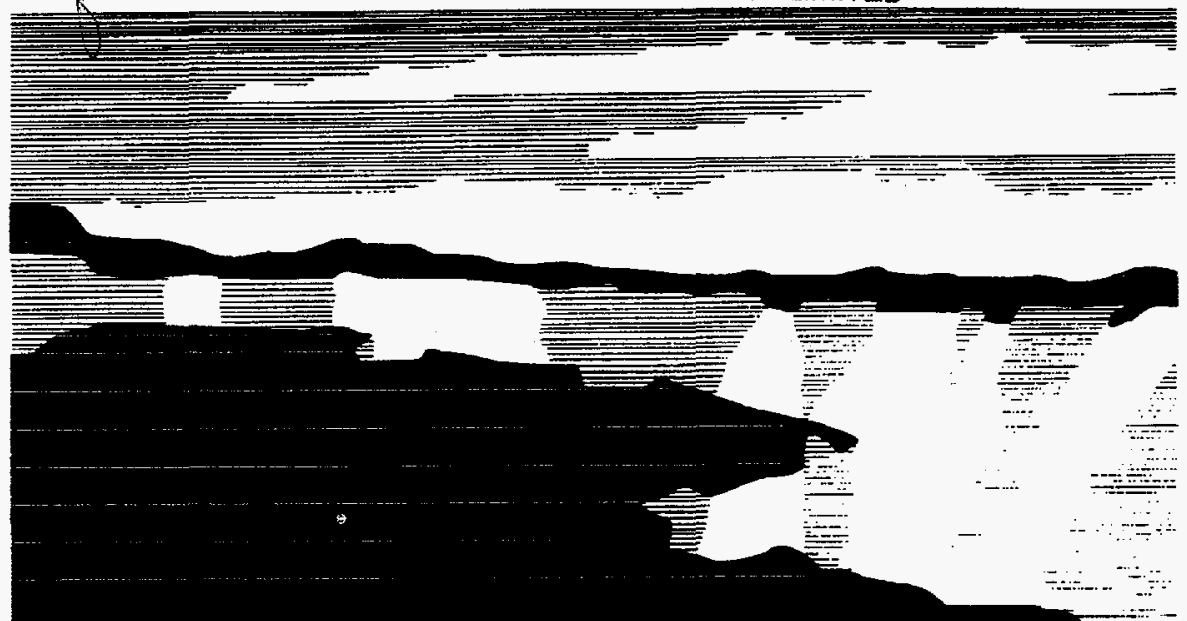

Los Alamos National Laboratory, an affirmative action/equal opportunity employer, is operated by the University of California for the U.S. Department of Energy under contract W-7405-ENG-36. By acceptance of this article, the publisher recognizes that the U.S. Government retains a nonexclusive, royaltyfree license to publish or reproduce the published form of this contribution, or to allow others to do so, for U.S. Govemment purposes. The Los Alamos National Laboratory requests that the publisher identify this article as work performed under the auspices of the U.S. Department of Energy. 


\section{DISCLAIMER}

This report was prepared as an account of work sponsored by an agency of the United States Government. Neither the United States Government nor any agency thereof, nor any of their employees, makes any warranty, express or implied, or assumes any legal liability or responsibility for the accuracy, completeness, or usefulness of any information, apparatus, product, or process disclosed, or represents that its use would not infringe privately owned rights. Reference herein to any specific commercial product, process, or service by trade name, trademark, manufacturer, or otherwise does not necessarily constitute or imply its endorsement, recommendation, or favoring by the United States Government or any agency thereof. The views and opinions of authors expressed herein do not necessarily state or reflect those of the United States Government or any agency thereof. 


\section{DISCLAIMER}

Portions of this document may be illegible in electronic image products. Images are produced from the best available original document. 


\title{
Separations Technology Development to Support Accelerator-Driven Transmutation Concepts
}

Francesco Venneri,* Edward Arthur, Charles Bowman, Roberto Camassa, Robert Ecke, Reed Jensen, Ning Li, Brian Newnam, and Mark Williamson

\begin{abstract}
This is the final report of a one-year Laboratory-Directed Research and Development (LDRD) Project at the Los Alamos National Laboratory (LANL). This project investigated separations technology development needed for accelerator-driven transmutation technology (ADTT) concepts, particularly those associated with plutonium disposition (accelerator-based conversion, $\mathrm{ABC}$ ) and high-level radioactive waste transmutation (accelerator transmutation of waste, ATW). Specific focus areas included separations needed for preparation of feeds to $A B C$ and ATW systems, for example from spent reactor fuel sources, those required within an $A B C / A T W$ system for material recycle and recovery of key long-lived radionuclides for further transmutation, and those required for reuse and cleanup of molten fluoride salts. The project also featured beginning experimental development in areas associated with a small molten-salt test loop and exploratory centrifugal separations systems.
\end{abstract}

\section{Background and Research Objectives}

Accelerator-driven transmutation technology (ADTT) systems are being defined and evaluated by Los Alamos National Laboratory as a means to deal with material legacies that have resulted, and will continue to result, from operation of nuclear facilities (both defense and civilian nuclear power). Such ADTT systems focus on destroying, through the transmutation of one nuclear species to another, long-lived radionuclides such as plutonium, other actinides (neptunium, americium), and certain long-lived fission products such as technetium and iodine. These radionuclides are major contributors to concerns associated with the back end of the nuclear fuel cycle -- concerns that include safeguards and

\footnotetext{
* Principal investigator, e-mail: venneri@lanl.gov
} 
proliferation issues, inadvertent criticality conditions resulting from underground disposal, and radioactive dose potentially released from repository environments over time frames stretching a thousand or more years into the future. Separations technology development is necessary for a system such as ADTT to achieve its operational objectives in a manner that meets material-diversion, performance, and environmental attractiveness objectives.

This LDRD effort has focused on identification and limited testing of materials separations technology that have a significant potential to meet such objectives. As described in this report, we have developed flowsheets for ADTT system separations with following unique features:

- They remove, by electrochemical means in a lithium fluoride-beryllium fluoride medium, uranium and zirconium components of spent reactor fuel. The remaining mixture of plutonium, other actinides, lanthanides, and fission products is then introduced into the ADTT system for further separations and transmutation. The approach is extremely diversion and proliferation resistant since no separated plutonium streams occur in the front-end process and since efforts to specifically recover plutonium will result in serious degradation of the carrier fluoride salt.

- They allow straightforward recovery of actinide fuel components for their subsequent recycle and further burning in the ADTT blanket. This process involves small amounts of material flow and can be done in a continuous, "slip stream" environment. The process also involves use of physical processes such as electrowinning, distillation, etc. that produce minimal waste streams.

- They include novel approaches to separations, such as the use of centrifugal contactors, to minimize materials requirements and improve the efficiency of material removal steps involving liquid-metal reduction processes.

Under this LDRD project, we have also built, operated, and diagnosed a state-of-the art molten-salt loop which is used to study molten salt properties and flows under modern conditions for environmental impact minimization. Additionally, experiments with highspeed centrifuges provided data useful for predicting the separation efficiency of lanthanide fission products following transmutation.

\section{Importance to LANL's Science and Technology Base and National R\&D Needs}

The deliverable result from this effort is the identification and definition of credible separations that can address, at a beginning level, potential DOE and other customer concerns about performance, feasibility, and waste production issues associated with operation of ADTT systems. This project supports the Laboratory's core competencies in nuclear and 
advanced materials as well as earth and environmental systems. It also enhances LANL's ability to respond to initiatives involving accelerator-driven transmutation technologies.

\section{Scientific Approach and Results to Date}

\subsection{Fuel Preparation}

Uranium and zirconium are constituents present in large amounts in spent-fuel feeds to ADTT systems. Although these materials must be safely disposed of as waste, they do not contribute to the major issues associated with the back end of the nuclear fuel cycle identified earlier. In addition, removal of uranium from the rest of the feed to an ADTT system prevents the production of additional plutonium during the transmutation campaign. Finally, the presence of uranium and zirconium in large quantities in the ADTT system would seriously impact its effectiveness for transmuting long-lived radionuclides by the process of unwanted neutron absorption on uranium and zirconium.

Molten-salt hydrofluorination followed by uranium and zirconium electrowinning is the process being pursued at Los Alamos to accomplish the spent fuel preparation. This process uses favorable differences in electrochemical potential in the fluoride molten salt medium to extract uranium and zirconium. If successfully implemented, it will allow the extraction of uranium and zirconium from spent fuel without separation of plutonium and fission products, thereby creating a robust-against-plutonium-diversion stream for introduction into ADTT.

The process consists of three main steps as shown in Figure 1. In the first step, the spent fuel assembly is chopped and immersed in molten fluoride salt, similar to the carrier salt used for the ADTT blanket. In the second step, $\mathrm{HF} / \mathrm{H}_{2}$ is sparged in the salt, so that fluorides are produced. The fluorides dissolve in the molten salt $\mathrm{Li}_{2} \mathrm{BeF}_{4}$ carrier. In the third step, when a beryllium anode is inserted in the molten salt, all of the fluoride species that are less stable than $\mathrm{BeF}_{2}$ will be reduced at the cathode. Beryllium lies between uranium and all the other actinides on the electrochemical potential scale as shown in Figure 2. Therefore, after the deposition of the metals and the uranium, all of the beryllium would have to come out of solution before any of the actinides, including plutonium, or the rare earth elements could be isolated. The composition of the salt mixture, after the uranium has been extracted, typically will have a 74:24:2 ratio of lithium fluoride to beryllium fluoride to actinide (plus fission product) fluorides.

The presence of beryllium is a strong barrier to the extraction of actinides or rareearth fission products during the spent-fuel preparation step. The species that remain in the molten salt will be $\mathrm{Np}, \mathrm{Pu}, \mathrm{Am}, \mathrm{Cm}$, and lanthanide fluorides, which can be fed directly into 
the molten salt blanket. Fission products (rare-earth elements) stay in solution with the actinides in the ADTT feed (they are removed subsequently within the ADTT system).

This process is sufficiently robust so that it could be used to deal with a variety of spent fuel forms. It integrates the process for removal of uranium and fuel clad materials with that needed to prepare the fuel mixture for the ADTT system. Because of its uniqueness -- method of separation, proliferation resistance, simplicity --we have put forward a disclosure of invention for the process.

\subsection{Fuel Cleanup Processes}

During ADTT operation, the by-products resulting from fission of the actinide fuel (fission products) must be removed from the carrier salt. Actinide residuals that remain in the carrier salt are then recycled to the system for further destruction through fission. Fission products in molten salt can be divided in three broad categories, and for each of these there is an appropriate means of cleanup. Volatile fission products and noble gases have low boiling points and tend to come out of the molten salt easily. Removal of these volatile species and noble gases from the molten salt is obtained by helium flow or sparging. Noble and seminoble metals have low solubility in the salt and will plate out on internal metal surfaces. The process was observed at Oak Ridge in their molten salt reactor experiment, and we plan to exploit it actively with the incorporation of passive extraction cells in the molten-salt flow path.

The remainder of the fission products, including the rare-earth elements, have good solubility in the salt and will tend to build up in concentration and stay in solution. Removal of lanthanides and other non-volatile species will be done using the lithium-bismuth reduction process, possibly followed by liquid centrifugation and precipitation. Whereas the first two processes could be performed continuously, the removal of the lanthanides will be done periodically. The lanthanides will accumulate in the fuel and be removed at 5- to 10year intervals, depending on the fuel used in the blanket. The point should be emphasized that the ADTT back-end processing targets fuel cleanup from fission products and does not allow actinide isolation and extraction. We now describe in more detail the main separation process components introduced above.

\section{2.a. Helium Sparging of Volatile Fission Products}

Since volatile fission products come out of the molten salt fuel naturally, the presence of a free surface above the salt allows the volatiles to escape. The efficiency for removal can be improved by sparging, that is by injecting helium bubbles in the molten salt stream. These bubbles act as collectors of the gaseous fission products. After extraction from the blanket, 
the helium stream carrying the fission products undergoes fractional distillation processes to extract the different fission products at different temperatures according to their boiling point. If extraction from the blanket is performed promptly, there is the possibility of achieving separation of cesium isotopes from the fractional decay of xenon.

\section{2.b. Electrolytic Separation of Noble and Semi-Noble Metals}

Electrochemical separation techniques are proposed for removing the "noble-metal" fission products: $\mathrm{Mo}, \mathrm{Nb}, \mathrm{Ru}, \mathrm{Rh}, \mathrm{Ag}, \mathrm{Cd}$, etc. The electrochemical method has been used extensively in other applications to produce pure metals from oxide- or halide-feed materials that have been dissolved in a molten salt. In this application, one is interested in purifying the molten salt rather than producing a pure metal.

An electrochemical cell would be placed in a region where flow of the fuel salt would occur. The electrochemical cell consists of a consumable anode, $\mathrm{Zr}$ or $\mathrm{Be}$, which is placed in the molten salt, and the "noble metals" are deposited on the cathode, a Ni rod. The fissile material, other actinides, and lanthanides remain in the molten salt. The reaction that describes the process is

$$
n \mathrm{Zr}(\mathrm{s})+4 \mathrm{MF}_{\mathrm{n}}(\mathrm{d})=\mathrm{n} \mathrm{ZrF}_{4}(\mathrm{~d})+4 \mathrm{M} \text { (s), }
$$

where $\mathrm{M}$ is a "noble metal", $\mathrm{n}$ is the valence state of the "noble metal", and $\mathrm{d}$ refers to a fluoride species dissolved in molten $\mathrm{Li}_{2} \mathrm{BeF}_{4}$. The reaction is spontaneous because of the free energy difference between $\mathrm{ZrF}_{4}$ and the "noble metal" fluorides. In principle, the electrochemical cell can be operated in a passive fashion, i.e., with no external source, although its efficiency can be enhanced by application of an external voltage. This system could also provide a dynamic method for control of the fluoride potential in the molten salt so that corrosion of the system's vessel could be limited.

Maintenance of this system would involve the periodic, remote replacement of the anode and cathode. The metals collected on the cathode would be oxidized, blended in silica, placed in glass, and sent to a storage facility.

\section{2.c. Lithium-Bismuth Reduction for Removal of Fission-Product Fluorides}

The process of lithium reduction in liquid bismuth was a key component of the fuel processing scheme for the Oak Ridge National Laboratory (ORNL) molten-salt, breederreactor concept. The ORNL exploited the different solubilities of lanthanides and actinides in bismuth to perform a separation of the two classes of elements using a multistage arrangement. In this process, the molten salt is allowed to come in contact with a 
lithium-bismuth solution. Depending on the amount of lithium present in the solution, a certain fraction of actinides and lanthanides will be reduced from the salt and dissolve into the bismuth. Typically, the actinides will tend to enter the bismuth solution first, so that by using several stages, an effective separation from the lanthanides can be achieved. The actinides are then collected and back-extracted into the salt.

The version adopted for ADTT applications is a simplified version of the ORNL process. We use a two-stage reduction process followed by back extraction as described above and shown in Figure 3. In the first step, the lithium concentration is selected to preferentially extract plutonium and other actinides into the bismuth phase. The lithium concentration is then adjusted so that the lanthanides are removed from the salt and transferred into a second bismuth phase. Afterwards, the actinides are back-extracted from

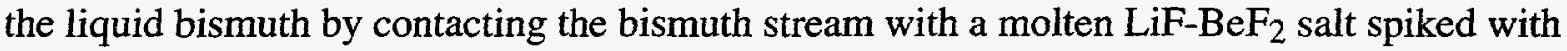
$\mathrm{BiF}_{3}$. Lanthanides left in the second bismuth stream could be removed by decreasing the temperature of the bismuth and collecting the solids that are formed. The bismuth present in each of the two streams would be recycled for further use.

\section{2.d. Centrifugation-Based Separation of Lanthanides from Actinides in a Bismuth Medium of Fission Products}

We have studied the use of centrifugation to enhance the performance of the separations of lanthanides, fission products, and actinides in a molten bismuth carrier. Molecular centrifugation uses the difference in molecular weight between the carrier (bismuth), fission products and actinides to obtain effective separations of the soluble fission products (including the lanthanides). Subjecting a liquid solution to large centrifugal fields will generate gradients of concentration increasing toward the outer edge of the spinning centrifuge rotor. These gradients, and the speed at which they are attained, are directly proportional to the square of the rotational velocity of the centrifuge and to the molecular weight of the solutes, relative to the carrier. Other factors such as the solution viscosity and temperature influence the process.

In the liquid bismuth solution coming out of the reduction step, described above, the lanthanides (lighter than the carrier) will develop a radial concentration gradient peaked toward the rotation axis, the actinides (heavier than the bismuth carrier) will develop a gradient peaked toward the outer radius of the centrifuge. By extracting the fission products on axis, the process is capable of efficiently removing them from the solution, regardless of their chemical affinity to the actinides. Through a series of centrifugation stages, very high enrichments (more than 1000:1) of light-to-heavy or heavy-to-light solute can be reached. The separated fission products can be concentrated in a final centrifugation stage to reduce 
the waste volume. Laboratory experiments with surrogate materials are described below in Section 3.3.c.

\section{2.e. Fuel Salt Remediation}

At the end of operation of an ADTT plant, the used molten salt must either have its fission products removed so that it can be reused, or it must be converted into a form that is qualified for disposal. The process for restoration of the salt would use the techniques described in the previous sections. Conversion of the salt into a form for disposal could be done using either of two proposed methods.

The first is based on precipitation. In this scheme, the beryllium fluoride would be vacuum-distilled along with the volatile fluorides. Potassium chloride and sodium chloride would be added to the salt solution to maintain liquidity. $\mathrm{Na}_{2} \mathrm{O}$ would be added to convert remaining actinide and fission product (with the exception of cesium) fluorides to oxides. These oxides would fall to the bottom of the solution, allowing the fluorides to be decanted away. The oxides would be fed into a glass vitrifier. As remaining fluorides solidify, they can be ground and leached in water to remove the CsF. The CsF could then be immobilized in pillared clay or silicio-titanates before disposal in glass.

The second scheme, based on electro-disposition, would use a series of electrowinning cells to first remove the beryllium and then the residual actinides, lanthanides, $\mathrm{Sr}$, and $\mathrm{Cs}$ in succession from the molten salt. Beryllium and collected actinides could be recycled or reintroduced into a follow-on ADTT system. The fission products and lanthanides would be converted to oxides and disposed of in a glass matrix.

\subsection{Laboratory Experiments}

\section{3.a. Molten-Salt Test Loop and Diagnostics}

To resolve a range of molten-salt technology issues, one being materials compatibility, a stainless-steel recirculating test loop was constructed and operated with a non-hazardous, eutectic salt mixture of $61 \% \mathrm{LiF}-39 \% \mathrm{NaF}$ at temperatures above its $651^{\circ} \mathrm{C}$ melting point. Vacuum distillation to remove the water of crystallization from the salt mixture was found necessary to prevent excessive corrosion to the stainless steel over the several day operating periods. Thermocouples provided data on temperature and flow condition, and voltammetric systems were developed to sense the concentration and nature of the solutes. Future additions will include Raman or infrared spectroscopy to identify the many liquid and gas species present.

The electrical conductivity of the molten-salt eutectic was measured to be $1.12 \mathrm{ohm}^{-1}$ $-\mathrm{cm}^{-1}$, about $3 \%$ the value of stainless steel. This modest value permitted direct internal 
electrical heating of the salt to temperatures above its melting point by use of a low ac voltage ( 3 to 9 volts). Such application of direct heating in a reactor may be exploited to maintain the salt in the liquid state without depending on fragile wire heaters.

\section{3.b. Separation of Volatile Species}

As introduced previously in Section 3.2., one of the post-exposure fuel cleanup processes will include helium gas sparging to remove certain volatile, highly absorbing species such as $\mathrm{Xe}, \mathrm{Kr}$, and $\mathrm{I}_{2}$ from the transmuter area on a very rapid time scale. Using a surrogate liquid of carbonated water, we conducted experiments that demonstrated that $\mathrm{He}$ sparging increased $\mathrm{CO}_{2}$ evolution from the solution by at least a factor of 20 . Similar increases in molten salts are expected.

\section{3.c. Centrifugal Separations}

A series of centrifuges operated at speed is one option being considered for separation of lanthanide fission products out of a solution containing actinides and lanthanides in liquid bismuth. To explore the efficiency of centrifugation, a series of laboratory experiments with surrogate aqueous solutions of $\mathrm{HAuCl}_{4}-3 \mathrm{H}_{2} \mathrm{O}, \mathrm{CsCl}$ and $\mathrm{NaCl}$ was conducted to determine the concentration profiles versus radial distance in the centrifuge as a function of time. Tests were conducted at 25,000 and $35,000 \mathrm{rpm}$ for as long as 136 hours. Periodic measurements of the concentration gradients agreed well with numerical simulations. The results serve to benchmark the codes that can be used for predicting the gradients at high temperature.

\subsection{Conclusions}

Techniques and processes to perform the required separation processes for ADTT systems have been identified and described. Considerable theoretical work has been devoted to the development of the techniques and the basic experimental work has begun on a welldiagnosed loop for studying properties of flowing molten salts. Additional experiments with

high-speed centrifuges provided data useful for predicting separation efficiency of lanthanide fission products following transmutation. As a result of this work, ADTT systems have a viable, self-consistent flow sheet that satisfies the most basic needs for simplicity and concerns for nuclear proliferation and material diversion.

\section{Publications:}

1. Li, Ning, Camassa, Roberto, Ecke, Robert, and Venneri, Francesco, Molecular Centrifugation of Liquid Solutions for Separation of Solution Constituents with Applications to Nuclear Spent Fuel Clean-up, LAUR-95-738, (1995). 
2. Li, Ning, Camassa, Roberto, Ecke, Robert, and Venneri, Francesco, Centrifugal Separation for Miscible Solutions: Fundamentals and Applications to Separation of Molten Salt Nuclear Materials, Proc. of Intl. Conf. on Accelerator-Driven

Transmutation Technologies and Applications, AIP Vol. 346, 799 (1995).

3. Li, Ning, Camassa, Roberto, Ecke, Robert, and Venneri, Francesco, Solutal Separation under Centrifugation, LAUR-95-1099 (1995).

4. Li, Ning, Camassa, Roberto, Ecke, Robert, and Venneri, Francesco, Decontamination of Nuclear Fuels with Centrifugal Separation, Proc. of Global 1995: Intil. Conf. on Evaluation of Emerging Nuclear Fuel Cycle Systems, Vol. 1, 1059 (1995).

5. Williamson, Mark A. and Venneri, Francesco, Front-End and Back-End Electrochemistry of Molten Salt in Accelerator-Driven Transmutation Systems, Proc. of Global 1995: Intl. Conf. on Evaluation of Emerging Nuclear Fuel Cycle Systems, Vol. 2, p. 1147 (1995). 


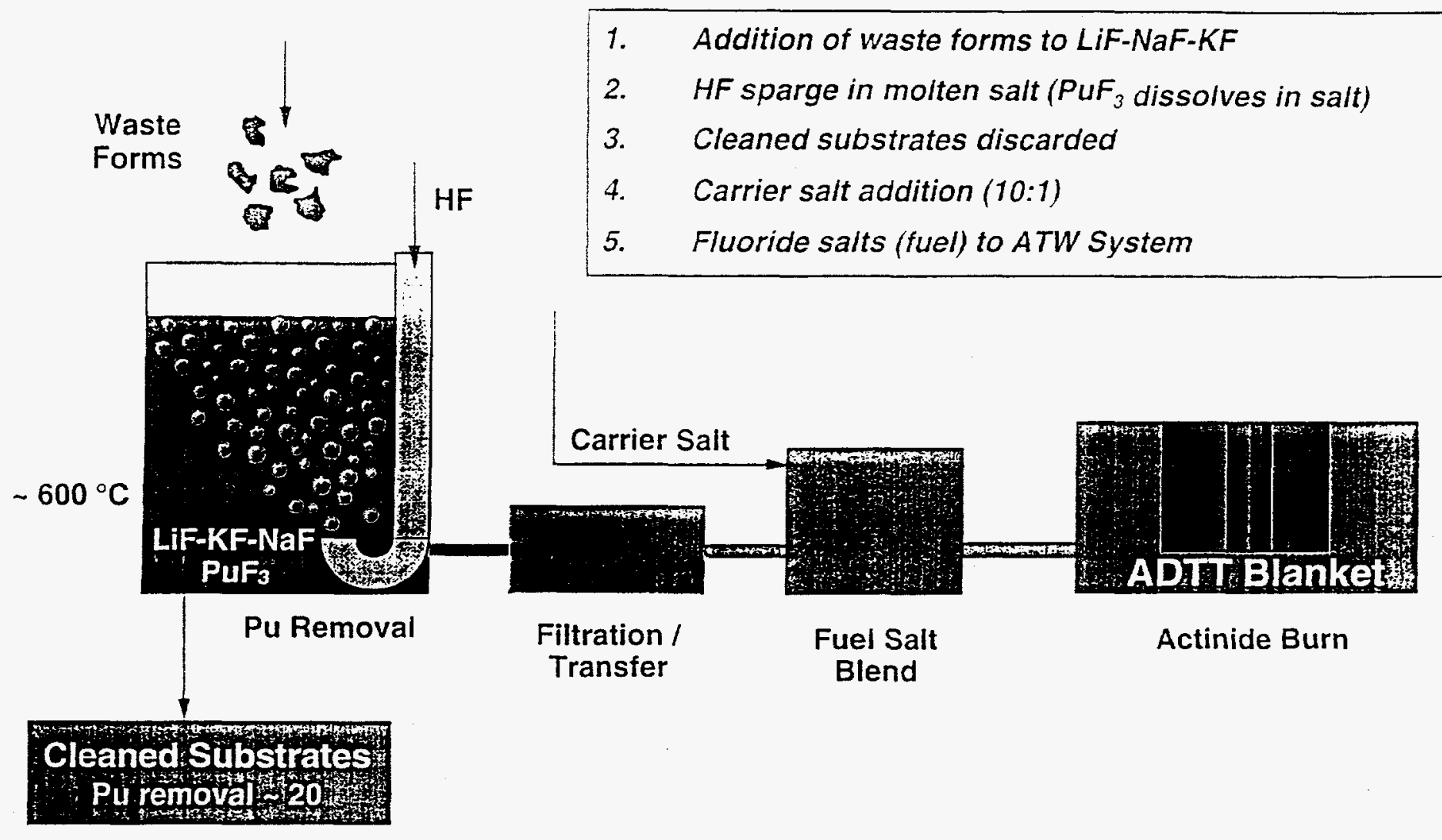

Fig.1 "Clean and Burn": the ADTT Treatment of Waste Forms

Fig. 1. Basis of electrowinning process for separating $\mathrm{U}^{\top}$ and $\mathrm{Zr}$ from actinides. 




Fig.2

Fuel Preparation for ADTT Systems

Fig. 2. Preparation of spent nuclear fuel for ADTT systems. 


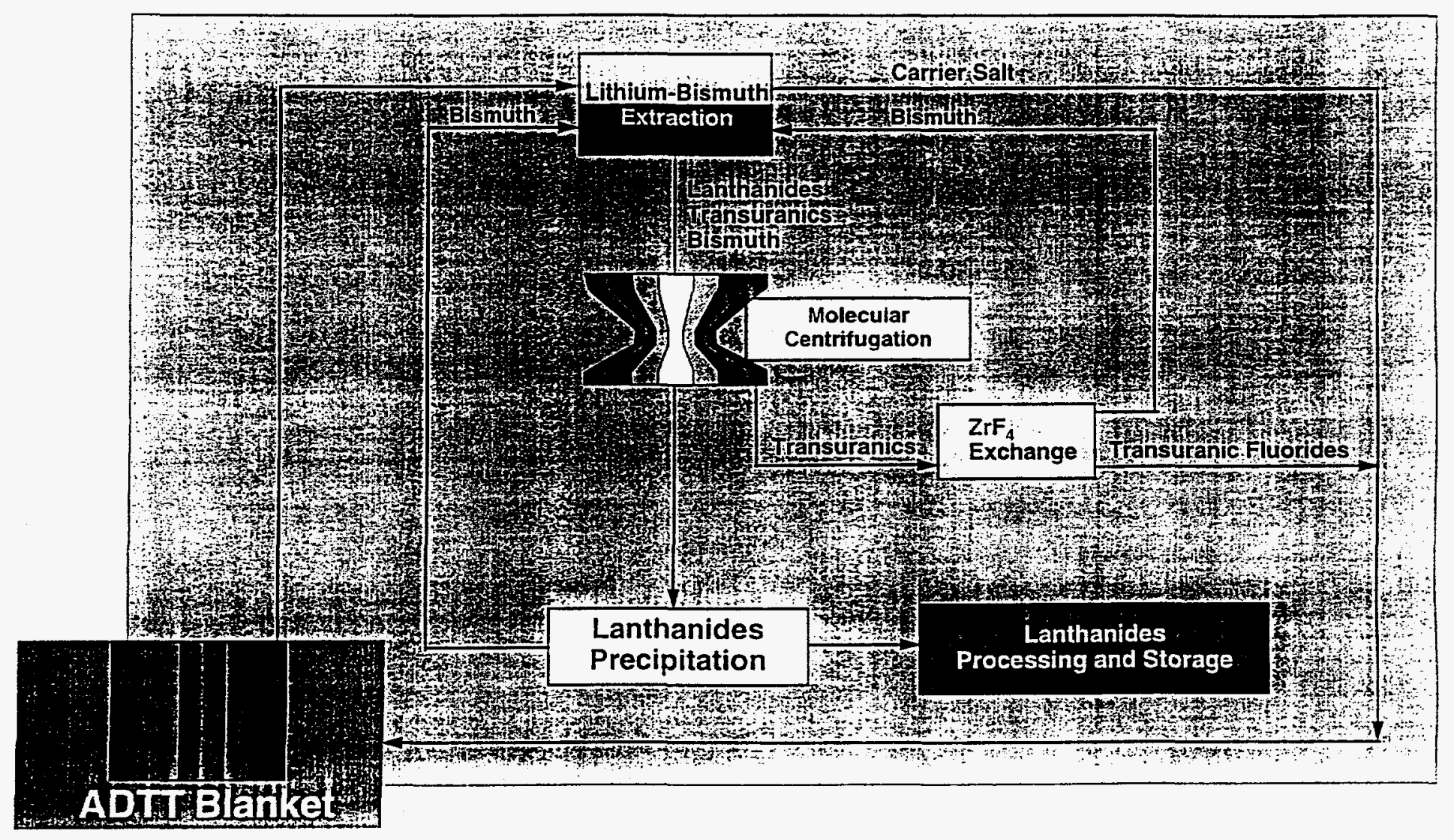

Fig 3. Lanthanides Separation Process

Fig. 3. Lathanide separation process for ADTT systems. 